\title{
Enhanced genome editing tools for multi-gene deletion knock-out approaches using paired CRISPR sgRNAs in CHO cells
}

Schmieder, Valerie; Bydlinski, Nina; Strasser, Richard; Baumann, Martina; Kildegaard, Helene Faustrup; Jadhav, Vaibhav; Borth, Nicole

\section{Published in:}

Biotechnology Journal

Link to article, DOI:

10.1002/biot.201700211

Publication date:

2018

Document Version

Peer reviewed version

Link back to DTU Orbit

Citation (APA):

Schmieder, V., Bydlinski, N., Strasser, R., Baumann, M., Kildegaard, H. F., Jadhav, V., \& Borth, N. (2018).

Enhanced genome editing tools for multi-gene deletion knock-out approaches using paired CRISPR sgRNAs in CHO cells. Biotechnology Journal, 13(3), [1700211]. https://doi.org/10.1002/biot.201700211

\section{General rights}

Copyright and moral rights for the publications made accessible in the public portal are retained by the authors and/or other copyright owners and it is a condition of accessing publications that users recognise and abide by the legal requirements associated with these rights.

- Users may download and print one copy of any publication from the public portal for the purpose of private study or research.

- You may not further distribute the material or use it for any profit-making activity or commercial gain

- You may freely distribute the URL identifying the publication in the public portal 
Research Article

ENHANCED GENOME EDITING TOOLS FOR MULTI-GENE DELETION KNOCK-OUT APPROACHES USING PAIRED CRISPR SgRNAS IN CHO CELLS ${ }^{\dagger}$

Valerie Schmieder ${ }^{1,2^{*}}$, Nina Bydlinski ${ }^{*}$, Richard Strasser ${ }^{1}$, Martina Baumann², Helene Faustrup Kildegaard ${ }^{3}$, Vaibhav Jadhav $2, \S$, Nicole Borth $1, \S$

${ }^{1}$ BOKU University of Natural Resources and Life Sciences, Vienna, Austria

${ }^{2} \mathrm{ACIB} \mathrm{GmbH}$, Austrian Center of Industrial Biotechnology, Graz, Austria

${ }^{3}$ The Novo Nordisk Foundation Center for Biosustainability, Technical University of

Denmark, Kgs. Lyngby, Denmark

*These authors contributed equally to this publication

\$Correspondence: Nicole Borth

BOKU University of Natural Resources and Life Sciences

Muthgasse 18, 1190 Vienna, Austria

nicole.borth@boku.ac.at

Vaibhav Jadhav

Austrian Center of Industrial Biotechnology GmbH

Muthgasse 11, 1190 Vienna, Austria

vaibhav.jadhav@acib.at

${ }^{\dagger}$ This article has been accepted for publication and undergone full peer review but has not been through the copyediting, typesetting, pagination and proofreading process, which may lead to differences between this version and the Version of Record. Please cite this article as doi: [10.1002/biot.201700211].

This article is protected by copyright. All rights reserved

Received: June 23, 2017 / Revised: August 31, 2017 / Accepted: September 29, 2017 
Keywords: CHO, CRISPR, bicistronic sgRNAs, genomic deletion, library

Abbreviations: CHO, Chinese Hamster Ovary; CRISPR, clustered regularly interspaced short palindromic repeat; sgRNA, single guide RNA; FUT8, $\alpha-1,6$-Fucosyltransferase 8; B4GALT, $\beta$-1,4-Galactosyltransferase; DSB, double stand break; NHEJ, non-homologous end-joining; InDel, insertion or deletion; LOF, loss-of-function; AsCpf1, Acidaminococcus_sp_BV3L6Cpf1; LbCpf1, Lachnospiraceae_bacterium_ND2006 Cpf1; GUSB, $\beta$-Glucuronidase; gDNA, genomic DNA; F-LCA, Fluorescein labeled Lens Culinaris Agglutinin; NucRed, NucRed® Live 647 ReadyProbes $® ;$ FACS, fluorescence activated cell sorting; cDNA, copy DNA; RT-qPCR, Reverse Transcription qPCR; DIG, Digoxigeninlabeled; GAPDH, Glyceraldeyde-3-phoshate Dehydrogenase; PAM, protospacer adjacent motif 


\section{Abstract}

Since the establishment of clustered regularly interspaced short palindromic repeat (CRISPR)/Cas9, powerful strategies for engineering of $\mathrm{CHO}$ cell lines have emerged. Nevertheless, there is still room to expand the scope of the CRISPR tool box for further applications to improve $\mathrm{CHO}$ cell factories.

Here, we demonstrate activity of the alternative CRISPR endonuclease Cpf1 in CHO-K1 for the first time and that it can be used in parallel to CRISPR/Cas9 without any interference. Both, Cas9 and Cpf1, can be effectively used for multi-gene engineering with a strategy based on paired single guide RNAs (sgRNAs) for full gene deletions. This strategy also enables the targeting of regulatory regions, which would not respond to the conventional frameshift mutations, as shown by deleting the $\alpha-1,6$-Fucosyltransferase 8 (FUT8) promoter resulting in a functional knock-out. Fut8 also served as model to verify that deletion efficiency is size-independent $(2-150 \mathrm{~kb})$.

To test the efficiency of multi-gene approaches in combination with gene deletion, clones harboring triple deletions in $\beta-1,4$-Galactosyltransferase (B4GALT) isozymes were identified using solely conventional PCR/qPCR. In addition two bicistronic transcription strategies were implemented to enable unequivocal pairing of sgRNAs: a CHO-derived tRNA linker that works for both, Cas9 and Cpf1, as well as paired sgRNAs in an array format, which can be used with Cpf1 due to its RNA processing ability.

These strategies broaden the range of application of CRISPR for novel gene editing approaches in $\mathrm{CHO}$ cells and also enable the efficient realization of a genome-wide deletion library. 


\section{Introduction}

Chinese Hamster Ovary (CHO) cells are the most widely used expression system for the production of complex therapeutic proteins since their establishment as cell factories in 1987 [1]. Despite the importance of CHO cells for industry, it was only recently that genomic and transcriptomic data of high quality was acquired [2-4]. However, now, that such data sets are available, the focus of optimization of cell performance has shifted from the original approach of optimizing media, cell selection procedures and production process conditions, towards directed cell engineering [5].

In parallel, the development of tools for targeted cell engineering has made a tremendous leap forward with the introduction of nucleases derived from bacterial clustered regularly interspaced short palindromic repeat (CRISPR) immune systems. Cas9, originally identified as the effector endonuclease of Streptococcus pyogenes [6], has been proven to be an efficient editing tool in many mammalian cells using a single chimeric RNA guide (single guide RNA - sgRNA) [7]. Cas9 introduces DNA double strand breaks (DSB), which are repaired by either homology directed repair or non-homologous end-joining (NHEJ), with the latter being the more frequent mechanism in mammalian cells [8]. NHEJ usually results in the insertion or deletion (InDel) of nucleotides at the targeted site. With CRISPR/Cas9, genes can be disrupted in a low-cost and time-saving manner, while ensuring high specificity for a large panel of targets [7]. Therefore, it is now possible to bring cell engineering to the next level: by combining numerous deletions of protein-coding regions and non-coding regulatory elements, entire cellular pathways can be either altered or completely blocked.

Cas9 has already been used quite extensively in CHO cells $[9,10]$ and further development of CRISPR based tools is happening at a rapid pace. Cpf1, a CRISPR class 2 type $\mathrm{V}$ enzyme, was recently introduced as an alternative editing system [11]. Two Cpf1 variants derived from Acidaminococcus_sp_BV3L6 (AsCpf1) and Lachnospiraceae_bacterium_ND2006 
(LbCpf1) were already found to be active in human cell lines [12]. Published work based on this novel system is limited and to our knowledge, its efficient use in CHO cells has not yet been demonstrated. Similar to Cas9, Cpf1 is an endonuclease guided by short RNA sequences and has an additional RNase activity [13]. Furthermore, Cpf1 has divergent properties in terms of recognition sequence, exact site of cutting and length of sgRNA. Due to these differences Cpf1 further broadens the range of possible applications for targeted engineering [14]. Specific characteristics of Cpf1 in comparison to the features of Cas9 are summarized in Tab. 1.

In most previous publications CRISPR/Cas9-mediated gene disruptions were introduced within the coding region of the targeted protein via a single sgRNA $[15,16]$. Hence, gene disruption is mostly based on frameshift mutations, which will statistically occur in only two out of three cases [17]. Usually sites for targeting are chosen that ensure loss-offunction (LOF): they are either located close to the translational start site or within a sequence that encodes for a crucial domain of the protein [16]. However, this approach raises several potentially problematic issues including altered transcripts or splicing variants, which could compensate or change gene function, as well as the accumulation of incomplete protein or of (frame-shifted) nonsense protein sequences [17]. Thus, we believe that to study a protein's role in a chosen system, it is necessary to remove the protein, which can only be ensured if the entire gene and thus its expression is completely eliminated as has been done by Hu et al. to investigate C-terminal lysine cleavage in antibody-producing CHO cells [18].

To achieve such complete removal of genes of interest, a combination of two sgRNAs needs to be transfected, leading to the loss of the intervening DNA sequence [19-22]. Although, the correlation between deletion frequency and deletion size was examined in human cells extensively reaching over one megabase, the link between genomic deletion range and the corresponding efficiency has not yet been evaluated in CHO $[17,21]$. To efficiently use such 
an approach, especially in the context of multiple gene knockouts in the same cell, the establishment of strategies for bicistronic transcription of sgRNA pairs is of advantage. Such strategies have been introduced with expression vectors using various linkers including tRNAs, RNA-triple-helix, introns, microRNAs and ribozyme sequences [23-25]. Bicistronic transcription with tRNAs has several benefits over other systems: the sequence is rather short and most importantly, post-transcriptional processing is efficiently conducted by the cell's endogenous machinery. The RNase activity of Cpf1 may turn out to be specifically beneficial in this context, as the autologous cleavage of sgRNAs allows further reduction of the overall length of bicistronic constructs as no linker regions are required [26]. Thus, such a strategy could be used for the generation of a genome-wide knock-out library, where the corresponding sgRNA pairs need to be tightly linked: the shorter the sequence to be synthesized for the library, the simpler it is to design and the more cost-efficient.

The following study aims to evaluate additional tools for CHO cell genome editing, with a view of enabling just such a genome-wide deletion library. Towards this end, the efficiency of such deletions was tested to be size-independent and the possibility to target also noncoding regions of the genome was evaluated by excising the promoter region of FUT8. Furthermore, different bicistronic sgRNA transcription strategies for Cas9 and AsCpf1 were established.

This paired sgRNA strategy allows screening for deletions by conventional PCR and abolishes the need for sequencing or other time-consuming methods for initial cell selection, thus enabling rapid and efficient selection of cell lines harboring multiple deletions.

\section{Materials and methods}

All gRNAs, gBlocks, primers for PCR/qPCR reactions and vector construction as well as probes used in Northern Blot analysis are listed in supporting information. 


\subsection{Cloning of gRNAs into CRISPR/Cas9 vector}

The vector pX458 (pSpCas9(BB)-2A-GFP, \#48138, Addgene) was used for introducing modifications in CHO-K1 cells mediated by Cas9 [7]. This plasmid allows expression of Cas 9 and additionally encodes for a second cassette with a cloning backbone for sgRNA expression from a U6 promoter. gRNAs with a length of 20 nt were designed with the help of the CRISPR guide online tool provided by Benchling and selected based on the probability of off-targets [27] and their predicted on-site activity [28]. The selected guide sequences were cloned into the pX458 vector as described by Bauer et al. [22].

\subsection{Construction of vectors for CRISPR/Cpf1 and cloning of gRNAs}

All-in-one plasmids were generated for AsCpf1 and LbCpf1. pY010 (pcDNA3.1-hAsCpf1, \#69982, Addgene) and pY016 (pcDNA3.1-hLbCpf1, \#69988, Addgene) served as backbones. The U6 promoter with two downstream BsmBI and flanking Pcil sites was amplified from pX458 using Phusion High-Fidelity DNA Polymerase (Thermo Fisher Scientific) with a three-step PCR followed by a two-step PCR. The amplified U6-BsmBI unit was inserted via Pcil into pY010 and pY016. Additionally, Gibson Assembly reaction was performed to clone T2A-GFP derived from pX458 into the BamHI restriction site of modified pY010 and pY016. The NEBuilder® HiFi Assembly Cloning Kit, containing a Q5® HighFidelity DNA Polymerase, was used for this purpose. Newly generated all-in-one plasmids are referred to as pY010(AsCpf1) and pY016(LbCpf1).

AsCpf1 and LbCpf1 gRNA sequences of 23 nt were designed as described in section 2.1. Chosen gRNAs with the scaffold sequence (21 nt) at the 5'-end and a 3'-Poly(T) tail were cloned according to Bauer et al. into the Cpf1 plasmids using BsmBI.

\subsection{Cell culture and transfection}


CHO-K1 cells were routinely cultivated in CD-CHO medium (Thermo Fisher Scientific) supplemented with $8 \mathrm{mM}$ Glutamine (Sigma-Aldrich) and 0.2\% Anti-Clumping Agent (Thermo Fisher Scientific). Cells were grown in either $125 \mathrm{ml}$ shaking flasks (Corning) with a working volume of $25-30 \mathrm{ml}$ or in $50 \mathrm{ml}$ spin bioreactor tubes (Techno Plastic Products) in $5-10 \mathrm{ml}$ medium. Shaking flasks and spin tubes were incubated at $37^{\circ} \mathrm{C}, 7 \% \mathrm{CO}_{2}$ and humidified air at a shaking speed of $140 \mathrm{rpm}$ and $250 \mathrm{rpm}$, respectively.

Nucleofection was done with a Neon® Nucleofector and the $100 \mu$ l Neon® Transfection Kit (Thermo Fisher Scientific) with $1700 \mathrm{~V}$ and 1 pulse of $20 \mathrm{~ms}$. Typically, 5 x $10^{6}$ cells in exponential growth phase were spun down and resuspended in $100 \mu$ r resuspension buffer containing 10 - $30 \mu \mathrm{g}$ endotoxin-free plasmid DNA. CHO-K1 cells transfected with TE buffer (mock) or the appropriate amount of the empty CRISPR all-in-one vector lacking a specific gRNA sequence (scramble) were used as controls/references for downstream analysis. Transfection efficiencies were determined one or two days' post transfection by flow cytometry (Gallios, Beckman Coulter) measuring the percentage of GFP-expressing cells. 1 x $10^{4}$ cells were analyzed using a $488 \mathrm{~nm}$ argon laser with a 525/50 filter. A minimum of 70\% GFP positive cells was expected.

\subsection{Determination of sgRNA activity}

The activity of individual sgRNAs was tested by detecting introduced InDel mutations using the Surveyor® Mutation Detection Kit (Integrated DNA Technologies). Genomic DNA (gDNA) was isolated on day four post transfection with the DNeasy® Blood \& Tissue Kit (Qiagen) according to the manufacturer's protocol. For the first step of the Surveyor $®$ assay the targeted genomic sites were amplified using Phusion High-Fidelity DNA Polymerase (Thermo Fisher Scientific). The mismatch detecting Surveyor ${ }^{\circledR}$ Nuclease assay was performed according to the manufacturer's protocol. The degree of InDel formation was determined via evaluation of DNA fragmentation by agarose gel electrophoresis using 
Midori Green Advance (Biozym) for DNA staining and ImageQuant ${ }^{\mathrm{TM}}$ for visual analysis. Relative InDel rates were calculated as described by Zetsche et al., 2015 [12].

\subsection{Crosstalk between CRISPR/Cas9 and CRISPR/Cpf1}

The U6-sgRNA sequences targeting FUT8 exon 2 for Cas9, AsCpf1 and LbCpf1 were PCR amplified with Phusion High-Fidelity DNA Polymerase (Thermo Fisher Scientific). PCR reactions were purified using the DNA Clean \& Concentrator ${ }^{\mathrm{TM}}-5$ from Zymo Research as recommended. Here, $1 \times 10^{6} \mathrm{CHO}-\mathrm{K} 1$ cells were transfected either with $5 \mu \mathrm{g}$ of the all-inone plasmids as positive controls or co-transfected with CRISPR enzyme plasmids with different combinations of the U6-sgRNA PCR products with a minimal requirement of $35 \%$ GFP positive cells. Crosstalk between Cas9 and AsCpf1/LbCpf1 was investigated by Surveyor assay analysis.

\subsection{Detection of genomic deletion of FUT8}

The generated FUT8 deletions were analyzed on genomic level by non-deletion and deletion PCR [22] as well as qPCR. gDNA was isolated four days after co-transfection of CHO-K1 cells with the appropriate Cas9 sgRNA combinations (10 $\mu$ g each). Non-deletion PCR was routinely performed with GoTaq ${ }^{\circledR}$ G2 DNA Polymerase (Promega). For deletion PCRs, Phusion High-Fidelity DNA Polymerase (Thermo Fisher Scientific) was used with a maximum elongation time of $30 \mathrm{sec}$. Non-deletion as well as deletion PCR products were visualized on a 2\% TAE agarose gel stained with Midori Green Advance (Biozym).

Genomic deletion efficiencies were quantified by qPCR. Samples were measured in quadruplicates using the SensiFAST ${ }^{\text {TM }}$ SYBR ${ }^{\circledR}$ Hi-ROX Kit (Bioline) with 20 ng gDNA as template and a reaction volume of $20 \mu \mathrm{L}$. qPCRs were run on a Rotor-Gene qPCR cycler (Qiagen) with 40 cycles of amplification. Average fold changes in FUT8 gDNA level 
normalized to $\beta$-Glucuronidase (GUSB) and relative to the mock were determined with the

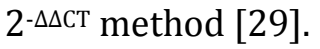

To analyze the phenotypic change of FUT8 deletion cell pools, Fluorescein-labeled Lens Culinaris Agglutinin (F-LCA, Vector Laboratories) staining in combination with flow cytometry (Gallios, Beckman Coulter) was performed one week after transfection. Additionally, cells were treated with NucRed ${ }^{\circledR}$ Live 647 ReadyProbes ${ }^{\circledR}$ (NucRed, Life Technologies) to detect live cells. $1 \times 10^{6}$ cells were washed once with PBS and incubated for $45 \mathrm{~min}$ at room temperature in the dark in complete medium containing $20 \mu \mathrm{g} / \mathrm{mL} \mathrm{F}$ LCA and two drops of NucRed. Cells were washed twice with complete medium before resuspension in it. $1 \times 10^{4}$ cells were analyzed by measuring green and red fluorescence using a $488 \mathrm{~nm}$ argon laser with a 525/50BP filter and a HeNe $633 \mathrm{~nm}$ laser with a 660BP filter.

\subsection{Generation of cell lines with multiple deletions in $\mathrm{N}$-Glycosylation genes}

For the generation of triple CHO-K1 knock-out cell lines, three subsequent rounds of transfection were performed, in which single genes were targeted by co-transfection of two pX458-sgRNA vectors using $15 \mu \mathrm{g}$ each. GFP positive cells were isolated by fluorescence activated cell sorting (FACS) on day 2 post transfection (MoFlo Astrios, Beckmann Coulter). Sorted cell pools were grown in cultivation medium supplemented with PenicillinStreptomycin (10.000 U/ml Penicillin, $10 \mathrm{mg} / \mathrm{ml}$ Streptomycin, VWR Chemicals) for three days. After expansion, cells were transfected with pX458 constructs targeting the next gene to be deleted.

Single clones were obtained by limiting dilution cloning using $50 \%$ conditioned medium. Cells were seeded in $200 \mu \mathrm{l}$ medium in 96 well plates at a cell density of two cells per well. Individual colonies were expanded in $900 \mu \mathrm{l}$ in 48 well plates and incubated at $37^{\circ} \mathrm{C}, 7 \%$ $\mathrm{CO}_{2}$ and humidified air at a shaking speed of $300 \mathrm{rpm}$. Samples for colony PCR were 
prepared by cell lysis in $20 \mu \mathrm{l} 0.2 \mathrm{M} \mathrm{NaOH}\left(10 \mathrm{~min}\right.$ at $75^{\circ} \mathrm{C}$, neutralization with $180 \mu \mathrm{l} 0.04$ M TRIS-HCl, $\mathrm{pH}$ 7.8) and subjected to three individual non-deletion PCR reactions for each gene. Lysates of selected clones were further evaluated by deletion PCR for all three genes. Total RNA of 1 - 5 x $10^{6}$ cells was isolated using TRI ${ }^{\circledR}$ reagent (Sigma-Aldrich, USA). Copy DNA (cDNA) for Reverse Transcription qPCR (RT-qPCR) was generated from 800 ng RNA using the High-Capacity cDNA Reverse Transcription Kit with RNase Inhibitor (Thermo Fisher Scientific) in reactions of $20 \mu \mathrm{l}$ according to the instructions provided.

cDNA was diluted 1:5 with nuclease-free water and RT-qPCRs were run in accordance to the protocol for gDNA qPCR. Here, $1 \mu$ diluted cDNA was used as template in $10 \mu \mathrm{L}$ reaction volume and Glyceraldeyde-3-phoshate Dehydrogenase (GAPDH) transcript levels served as internal control for normalization.

\subsection{Bicistronic sgRNA transcription}

Bicistronic sgRNAs containing the tRNA linker were ordered as gBlocks from Integrated DNA Technologies (IDT) with flanking cloning sites for both CRISPR systems. Arrayed AsCpf1 sgRNAs were ordered as single-stranded oligonucleotides with BsmBI overhangs from Sigma-Aldrich, which were then annealed and phosphorylated. The double-stranded AsCpf1 array as well as the tRNA gBlocks targeting FUT8 were inserted into the CRISPR allin-one plasmids (pX458 and pY010(AsCpf1)) with BsmBI and BbsI. Intracellular processing of bicistronically transcribed sgRNAs was investigated by non-radioactive Northern Blot. Total RNA from transfected cells was isolated two days post treatment. For each sample $20 \mu$ g RNA were resolved by a 10\% TBE-Urea-PAGE gel (Invitrogen) and blotted onto a Hybond-N+ nylon membrane (Roche). The membrane was cross-linked by ultraviolet light and hybridized overnight with Digoxigenin-labeled (DIG) probes (DIG Oligonucleotide Tailing Kit, 2nd generation, Roche). DIG Luminescent Detection Kit (Roche) was used for 
detection according to manufacturer's manual. Functionality of released Cas9 or AsCpf1 sgRNAs against FUT8 was determined by F-LCA staining eleven days post transfection.

\section{Results}

\subsection{Activity of CRISPR/Cpf1 in CHO-K1 and crosstalk with CRISPR/Cas9}

Initially, the two Cpf1 enzymes, AsCpf1 and LbCp1, were examined for their functionality in CHO. AsCpf1 and LbCpf1 all-in-one plasmids were generated containing a T2A-linked GFP transfection marker and a U6 promoter for expression of sgRNAs together with two BsmBI restriction sites (Fig. 1A). Next, overlapping gRNAs for Cas9 as well as for AsCpf1 and LbCpf1 targeting FUT8 exon 2 (Fig. 1B) were designed to verify Cpf1 activity and the functionality of the modified vectors. Relative InDel modification rates of $21 \%, 19 \%$ and 12\% were estimated for AsCpf1, LbCpf1 and Cas9 respectively (Fig. 1C). To enable multiple parallel deletion approaches that can be controlled independently of each other, potential crosstalk between the two Cpf1 enzymes and the Cas9 system was evaluated. Therefore, CRISPR enzyme expressing vectors were transfected together with U6-sgRNA PCR products for the different CRISPR systems in every possible combination (Fig. 1D). The all-in-one plasmids, already used for the analysis of AsCpf1 and LbCpf1 activity in CHO-K1, served as positive controls. The mock sample and each scramble control were used as negative controls (Fig. 1E). The respective positive controls as well as the suitable combinations of Cas9, AsCpf1 and LbCpf1 enzyme plasmid together with the corresponding U6-sgRNA PCR product resulted in detectable genome modifications (Cas9: 18\% and 9\%, AsCpf1: 27\% and 11\%, LbCpf1: 17\% and 10\%). There were no indications for the presence of DNA cleavage when using one of the CRISPR enzyme expressing plasmids in combination with one of the other CRISPR system's U6-sgRNA PCR products. AsCpf1 was selected for further development of the CRISPR tool box in CHO. 


\subsection{Evaluation of genomic deletion size and modification efficiency using co- transfected sgRNA pairs}

FUT8 was selected as a model gene to establish the strategy for generating LOF mutations by full genomic deletion of targeted gene using two CRISPR/Cas9 sgRNAs. At the same time the feasible genomic deletion range and the corresponding efficiency was evaluated. FUT8 is the only enzyme responsible for core $\alpha-1,6$-fucosylation in CHO-K1 cells both for secreted and surface proteins [2]. Therefore, it offers the possibility to easily determine the efficiency of deletions by monitoring the binding of a specific lectin to the cell surface. Six different CRISPR/Cas9 gRNAs were generated targeting untranslated parts of the gene and their functionality was evaluated via Surveyor assay analysis (Supporting information, Fig. S1). Depending on the combination of sgRNAs for co-transfection, genomic deletions ranging from approximately $2 \mathrm{~kb}$ up to $150 \mathrm{~kb}$ were covered. Additionally, a pair of sgRNAs was cloned to target the FUT8 promoter (Fig. 2A) as identified based on the CHO Epigenome database [30]. The workflow for the analysis of genomic deletions and their efficiencies is depicted in Figure 2B. The occurrence of genomic deletions was verified by amplifying the targeted regions for non-deletion and deletion PCR. A decrease in non-deletion PCR signal intensities was observed for all FUT8 deletion cell pools except when targeting the promoter, since the diagnostic primer pair is binding within the FUT8 gene. Relative intensities were determined ranging from 65 - $85 \%$ compared to the mock control. Furthermore, deletion PCR bands were obtained for all FUT8 modified samples (Fig. 2C). To quantify the reduction of intact gene copies in the pools, we determined the FUT8 gDNA fold change by qPCR (Fig. 2D). Here, the used primer pair was binding within the FUT8 gene and is therefore not applicable to determine the deletion efficiency for the FUT8 promoter sample. An average reduction of $0.30 \pm 0.05$ fold relative to the mock control was detected for all other deletion pools. The level of reduction was independent of the deletion size. 
Further, FUT8 LOF mutations were analyzed in the modified CHO-K1 cell pools using F-LCA staining (Fig. 2E; supporting information, Fig. S3). For the gene deletion samples between $40-60 \%$ of analyzed cells were F-LCA negative, indicating a significant reduction of FUT8 expression one week after transfection. Moreover, targeting the FUT8 promoter also resulted in a decrease of F-LCA positive cells of around 20\%. A comparable change in phenotype was not observed when using each sgRNA individually except for sg7, which is binding close to the approximate exon (Supporting information, Fig. S2 and Fig. S6).

\subsection{Generation of clones with multiple deletions in N-Glycosylation genes}

In the context of pathway engineering, it may be necessary to remove several genes to completely inactivate or modify a pathway. Likewise, in the context of isoenzymes it is often unclear what the precise functions and contributions of the individual enzymes are. This would require deleting several isoenzymes in parallel to leave a single isoform for in depth characterization. To evaluate our full deletion strategy for multiplexing, we chose three isoforms of B4GALT and targeted them by paired sgRNAs as depicted in Figure 3A. The fragments to be excised from the genome have a size of 18.3, 5.2 and $18.9 \mathrm{~kb}$ for B4GALT1, B4GALT2 and B4GALT4, respectively. Figure 3B shows the outline of the workflow. A deletion for each gene was introduced consecutively in three subsequent rounds of transfection and sorting for GFP expression. 144 clones obtained by single cell dilution were expanded and in the first round of screening, non-deletion PCR was performed for all three targets (Supplementary information, Fig. S3). At this stage many clones were already excluded as they still had at least one gene copy of the individual targets, resulting in a subset of 23 clones, which showed non-deletion and deletion PCR profiles suggesting full deletion of two $(n=21)$ or three genes $(n=2)$. Out of these 23 clones, 17 were selected for further investigation to obtain similar clone numbers for each combination. For clone characterization, RNA was isolated and RT-qPCR was performed to verify lack of expression 
of targeted galactosyltransferases to ensure LOF. Analysis by RT-qPCR resulted in identification of a total of three clones with no detectable expression of all three targets (Supplementary information, Fig. S4). One potential triple knock-out clone was proven to have been incorrectly suspected to carry three deletions, while two potential double knockout clones also lacked expression of the residual third target. Here analysis by non-deletion and deletion PCR suggest that both clones had one copy of B4GALT2 deleted, while the other was edited in a way that precludes transcription. Furthermore, five clones showed to have two genes deleted (B4GALT1/2 deletion: four clones, B4GALT1/4 deletion: one clone) and seven clones with either B4GALT1 or B4GALT2 fully knocked-out were identified (Fig. 3C). Thus, of all possible combinations for this set of paired sgRNAs, the only two missing after this approach was a B4GALT2/4 double and a B4GALT4 single deletion (Supplementary information, Fig. S4).

\subsection{Bicistronic sgRNA transcription for CRISPR/Cas9 and CRISPR/AsCpf1 in CHO-K1}

To permit sgRNA delivery of a defined pair into the same cell to induce a specific genomic deletion, which would be a prerequisite for the realization of a full genome deletion library, we implemented bicistronic sgRNA transcription strategies for Cas9 and AsCpf1 (Fig. 4A). To remain within the endogenous CHO system, a CHO tRNAGly sequence was used to link a specific sgRNA pair and to force their intracellular processing by endogenous RNase enzymes. Design of bicistronic sgRNA cassettes resulted in a total length of around $270 \mathrm{nt}$ (Cas9) and $165 \mathrm{nt}$ (AsCpf1), respectively. Additionally, we constructed an AsCpf1 sgRNA array system without any extra linker for Cpf1 RNase-mediated intracellular editing, thus reducing the bicistronic sgRNA length further to $86 \mathrm{nt}$. To verify RNA processing and to prove functionality of the released guides, sgRNAs to introduce $12.5 \mathrm{~kb}$ (Cas9) or $50.9 \mathrm{~kb}$ (AsCpf1) genomic FUT8 deletions were tested by transfection of CHO-K1 cells with plasmids containing either the linked sgRNA pair or with vectors expressing the same sgRNAs 
individually as positive control. Northern Blot analysis of total RNA using DIG- labeled probes against the Cas9 scaffold and the AsCpf1 sgRNA targeting FUT8 exon 2 were performed (Fig. 4B). In case of Cas9 a single band was observed for the two plasmid approach as well as for the sgRNA-tRNA system with a size of approximately $100 \mathrm{nt}$. The same results were obtained for both AsCpf1 bicistronic sgRNA transcription approaches, with bands at the expected size of around $45 \mathrm{nt}$. Next, the CRISPR enzyme guiding capability of intracellularly released sgRNAs for all three different paired transcription strategies was evaluated by F-LCA (Fig. 4C; supporting information, Fig. S6). For CRISPR/Cas9 a reduction of the F-LCA positive cell population by about $15 \% \pm 7 \%$ was observed when transfecting CHO-K1 cells with the two plasmid approach as well as the sgRNA-tRNA carrying plasmid. The spiked sample, which is composed of the scrambled plasmid and the sgRNA-tRNA vector to achieve similar molecular amounts of sgRNAs compared to the two plasmid approach, resulted in a reduction of F-LCA positive cells by around 5\%. Targeting FUT8 with AsCpf1 sgRNA pairs decreased the percentage of F-LCA positive cells by overall $14 \% \pm 5 \%$. Here, co-transfection of the separate sgRNA expressing plasmids induced a signal reduction of $8 \%$, while the sgRNA-tRNA approach spiked with the scrambled plasmid induced a $10 \%$ reduction. Both, the sgRNA-tRNA strategy as well as the sgRNA array for AsCpf1 lowered the percentage of F-LCA positive cells by $20 \%$, comparable to the Cas 9 sgRNA-tRNA approach. Additionally, functionality of the released sgRNA pairs, both for Cas9 and AsCpf1, was evaluated by deletion PCR and gDNA qPCR (Supporting information, Fig. S7).

\section{Discussion}

The establishment of precision genome editing tools such as CRISPR has led to a surge in engineering approaches that, because of the simplicity and efficiency of these methods, enable strategies, which were previously considered impossible due to the high work load. Such scenarios include genome-wide knock-out studies as well as multi-gene pathway 
engineering that targets several genes at the same time. However, to efficiently realize such strategies, the available tools require some optimization and fine-tuning. In this study, we propose several advancements that will help with the above.

The most prominent limitation in this respect is the current practice of aiming to achieve frameshifts of coding genes for LOF generation. In a standard protocol, cells are transfected with a sgRNA that typically targets an exon of a functional domain or close to the transcription start site followed by subcloning and screening for frameshift mutations by sequencing. Here, on the other hand we propose a full gene deletion strategy using two sgRNAs, which offers several advantages compared to the InDel formation strategy: i) Complete deletion of a gene results in predictability of LOF [22]. ii) The efficiency in generating bi-allelic knock-outs is high [17]. This was proven in our study simply by the fact that we were able to detect significant changes in FUT8 expression, since Yamane-Ohnuki et al. reported that a bi-allelic FUT8 knock-out is required to obtain a discoverable LOF modification [31]. iii) Standard PCR and RT-qPCR are used to determine whether a cell line contains no, a mono- or a bi-allelic knock-out of a gene. Thus, only two PCR techniques are adequate for the identification of successful deletions, which is cost efficient, fast and does not limit the number of clones that can be screened in parallel [19]. iv) Frameshift mutation strategies are not applicable in case of non-coding RNAs or regulatory DNA stretches, however, the deletion strategy can be applied also to study the function of such genomic regions. We have shown that such regulatory genome editing is possible by deleting the FUT8 promoter. v) Most importantly, in the context of knocking out multiple genes or entire pathways, we speculate that frameshift mutants would eventually lead to cells filled with multiple dysfunctional, probably misfolded proteins that may cause unknown side effects and stress responses could occur, which in turn are likely to impair the cell in other ways than those caused by the LOF. This may have significant effects on the interpretation of functional studies. 
To use such a paired sgRNA strategy for larger scale, multiplexed or genome-wide library driven engineering approaches, additional tools are necessary. First, the corresponding pair of sgRNAs needs to be transfected into a cell jointly, ideally derived from a single transcript, to ensure that the guides are not mixed up, which is of relevance especially in multiplexed or library approaches. Mainly for large-scale libraries the limiting factor is the size of the scaffold, which in case of Cas9 would lead to around 200 nt for a pair of sgRNAs, not including a linker sequence. Libraries of such length are not easily synthetized, as the efficient length of a nucleotide sequence that can be generated with precision is approximately $170 \mathrm{bp}$. Strategies to overcome this problem for paired CRISPR/Cas9 sgRNA libraries have been published but include additional cloning steps, which are more laborious and tend to have the disadvantage of increased mutation and recombination rates $[32,33]$. The use of the alternative CRISPR system Cpf1, which has a shorter scaffold and RNA editing abilities, enables the use of much shorter pairs of approximately $90 \mathrm{bp}$. So far, functionality of the alternative CRISPR enzyme Cpf1 from the two bacterial variants As and Lb was only proven in human cells and Drosophila [12,34]. We demonstrated efficient genome engineering by AsCpf1 and LbCpf1 in CHO-K1 for the first time. Since the established tools for paired Cas9 as well as AsCpf1 sgRNAs are either using the endogenous tRNA processing machinery [35] or the Cpf1 RNase activity [26] for intracellular processing of the bicistronic transcripts, no additional components have to be added to the system or transfected into cells and hence, offer an efficient approach for gene multiplexing or other applications. For each bicistronic sgRNA expression strategy correct intracellular editing was proven by Northern Blot analysis. No additional signals for unprocessed/incorrectly processed paired sgRNA transcripts were detected indicating that the majority of sgRNAs was properly released.

The verification of the applicability of this system for $\mathrm{CHO}$ has additional advantages: it enlarges the possible range of protospacer adjacent motif (PAM) sites to AT-rich regions 
(Cpf1 PAM is 5'-TTTN) [14], as use of Cas9 is partially restricted to GC-rich areas. Furthermore, it enables using CRISPR for two different intentions in the same cell without any crosstalk. For instance, in the above-mentioned library screening approach, one would want to be able to easily identify the region deleted in a given cell, which was selected for based on its advantageous properties. This would require that the sgRNA pair from the transfected library was integrated into a specific genomic locus, which would have the additional advantage of avoiding unspecified effects by gene disruption due to random integration. Thus, one would have to achieve targeted integration by CRISPR without at the same time allowing any genome editing of the transiently expressed sgRNAs from the library. To achieve this, Cas 9 could be used for integration, while Cpf1 could be used for the subsequent deletions by the integrated sgRNA pairs [36].

The fact that the size of the deleted region appears to be not limited within a large range of $2-150 \mathrm{~kb}$ enables the efficient generation of a genome-wide library of reasonable size. In contrast to the findings of Canver et al. [17], the modification rates for the genomic deletions performed in our study were size-independent and are hence, in all likelihood, more substantially determined by the genome engineering capability of the individual sgRNAs. Comparable observations were also published by Zheng et al.[19].

In summary, a competent strategy for the generation and screening of LOF mutants by inducing size-independent genomic deletions of targeted genes was demonstrated. Moreover, the CRISPR tool box was expanded for its application in $\mathrm{CHO}$ with alternative CRISPR/Cpf1 systems as well as bicistronic sgRNA delivery.

The establishment of these tools now enables the realization of a genome-wide CHO deletion library to screen for process-relevant properties that comprises both coding and non-coding regions of the genome. So far, it has not been attempted to perform a genetic screen of this scope for this important cell line. Previous knock-out libraries established in other cellular systems were focusing on the protein coding regions that cover only $3 \%$ of all 
genetic information $[37,38]$. We propose that it is now possible to aim for a coverage of the entire genome, which is of great relevance as enough evidence for the importance of functional non-coding regions has already accumulated. 


\section{Acknowledgement}

This work has been supported by the Austrian BMWFW, BMVIT, SFG, Standortagentur Tirol, Government of Lower Austria and Business Agency Vienna through the Austrian FFGCOMET- Funding Program. The authors gratefully acknowledge support by the FWF "Biotechnology of Proteins" PhD Program Grant \# W1224 and the "eCHO Systems" ITN PhD Program funded by the European Union's Horizon 2020 research and innovation program under the Marie Skłodowska-Curie grant agreement No 642663. Additionally, H. F. Kildegaard thankfully acknowledges support from the Novo Nordisk Foundation.

\section{Conflict of interest}

The authors declare no financial or commercial conflict of interest. 


\section{References}

[1] Walsh, G., Biopharmaceutical benchmarks 2014. Nat. Biotechnol. 2014, 32, 992-1000.

[2] Xu, X., Nagarajan, H., Lewis, N.E., Pan, S., Cai, Z., Liu, X., Chen, W., Xie, M., Wang, W., Hammond, S., Andersen, M.R., Neff, N., Passarelli, B., Koh, W., Fan, H.C., Wang, J., Gui, Y., Lee, K.H., Betenbaugh, M.J., Quake, S.R., Famili, I., Palsson, B.O., Wang, J., The genomic sequence of the Chinese hamster ovary (CHO)-K1 cell line. Nat. Biotechnol. 2011, 29, 735-741.

[3] Brinkrolf, K., Rupp, O., Laux, H., Kollin, F., Ernst, W., Linke, B., Kofler, R., Romand, S., Hesse, F., Budach, W.E., Galosy, S., Müller, D., Noll, T., Wienberg, J., Jostock, T., Leonard, M., Grillari, J., Tauch, A., Goesmann, A., Helk, B., Mott, J.E., Pühler, A., Borth, N., Chinese hamster genome sequenced from sorted chromosomes. Nat. Biotechnol. 2013, 31, 694695.

[4] Lewis, N.E., Liu, X., Li, Y., Nagarajan, H., Yerganian, G., O’Brien, E., Bordbar, A., Roth, A.M., Rosenbloom, J., Bian, C., Xie, M., Chen, W., Li, N., Baycin-Hizal, D., Latif, H., Forster, J., Betenbaugh, M.J., Famili, I., Xu, X., Wang, J., Palsson, B.O., Genomic landscapes of Chinese hamster ovary cell lines as revealed by the Cricetulus griseus draft genome. Nat. Biotechnol. 2013, 31, 759-765.

[5] Kim, J.Y., Kim, Y.-G., Lee, G.M., CHO cells in biotechnology for production of recombinant proteins: current state and further potential. Appl. Microbiol. Biotechnol. 2012, 93, 917930.

[6] Jinek, M., Chylinski, K., Fonfara, I., Hauer, M., Doudna, J.A., Charpentier, E., A Programmable Dual-RNA-Guided DNA Endonuclease in Adaptive Bacterial Immunity. Science 2012, 337, 816-821.

[7] Ran, F.A., Hsu, P.D., Wright, J., Agarwala, V., Scott, D.A., Zhang, F., Genome engineering using the CRISPR-Cas9 system. Nat. Protoc. 2013, 8, 2281-2308.

[8] Lee, J.S., Kallehauge, T.B., Pedersen, L.E., Kildegaard, H.F., Site-specific integration in CHO cells mediated by CRISPR/Cas9 and homology-directed DNA repair pathway. Sci. Rep. 2015, 5, 8572.

[9] Ronda, C., Pedersen, L.E., Hansen, H.G., Kallehauge, T.B., Betenbaugh, M.J., Nielsen, A.T., Kildegaard, H.F., Accelerating genome editing in CHO cells using CRISPR Cas9 and CRISPy, a web-based target finding tool. Biotechnol. Bioeng. 2014, 111, 1604-1616.

[10] Sun, T., Li, C., Han, L., Jiang, H., Xie, Y., Zhang, B., Qian, X., Lu, H., Zhu, J., Functional knockout of FUT8 in Chinese hamster ovary cells using CRISPR/Cas9 to produce a defucosylated antibody. Eng. Life Sci. 2015, 15, 660-666. 
[11] Yamano, T., Nishimasu, H., Zetsche, B., Hirano, H., Slaymaker, I.M., Li, Y., Fedorova, I., Nakane, T., Makarova, K.S., Koonin, E.V., Ishitani, R., Zhang, F., Nureki, O., Crystal Structure of Cpf1 in Complex with Guide RNA and Target DNA. Cell 2016, 165, 949-962.

[12] Zetsche, B., Gootenberg, J.S., Abudayyeh, O.O., Slaymaker, I.M., Makarova, K.S., Essletzbichler, P., Volz, S.E., Joung, J., van der Oost, J., Regev, A., Koonin, E.V., Zhang, F., Cpf1 Is a Single RNA-Guided Endonuclease of a Class 2 CRISPR-Cas System. Cell 2015, $163,759-771$.

[13] Fonfara, I., Richter, H., Bratovič, M., Le Rhun, A., Charpentier, E., The CRISPRassociated DNA-cleaving enzyme Cpf1 also processes precursor CRISPR RNA. Nature 2016, 532, 517-521.

[14] Kleinstiver, B.P., Genome editing: Showdown between Cas9 and Cpf1. Nat. Methods 2016, 13, 714-714.

[15] Grav, L.M., Lee, J.S., Gerling, S., Kallehauge, T.B., Hansen, A.H., Kol, S., Lee, G.M., Pedersen, L.E., Kildegaard, H.F., One-step generation of triple knockout CHO cell lines using CRISPR/Cas9 and fluorescent enrichment. Biotechnol. J. 2015, 10, 1446-1456.

[16] Chiu, J., Valente, K.N., Levy, N.E., Min, L., Lenhoff, A.M., Lee, K.H., Knockout of a difficult-to-remove $\mathrm{CHO}$ host cell protein, lipoprotein lipase, for improved polysorbate stability in monoclonal antibody formulations. Biotechnol. Bioeng. 2017, 114, 10061015.

[17] Canver, M.C., Bauer, D.E., Dass, A., Yien, Y.Y., Chung, J., Masuda, T., Maeda, T., Paw, B.H., Orkin, S.H., Characterization of Genomic Deletion Efficiency Mediated by CRISPR/Cas9 in Mammalian Cells. J. Biol. Chem. 2014, jbc.M114.564625.

[18] Hu, Z., Zhang, H., Haley, B., Macchi, F., Yang, F., Misaghi, S., Elich, J., Yang, R., Tang, Y., Joly, J.C., Snedecor, B.R., Shen, A., Carboxypeptidase D is the only enzyme responsible for antibody C-terminal lysine cleavage in Chinese hamster ovary (CHO) cells. Biotechnol. Bioeng. 2016, 113, 2100-2106.

[19] Zheng, Q., Cai, X., Tan, M.H., Schaffert, S., Arnold, C.P., Gong, X., Chen, C.-Z., Huang, S., Precise gene deletion and replacement using the CRISPR/Cas9 system in human cells. BioTechniques 2014, 57, 115-124.

[20] Zhang, L., Jia, R., Palange, N.J., Satheka, A.C., Togo, J., An, Y., Humphrey, M., Ban, L., Ji, Y., Jin, H., Feng, X., Zheng, Y., Large Genomic Fragment Deletions and Insertions in Mouse Using CRISPR/Cas9. PLoS ONE 2015, 10.

[21] He, Z., Proudfoot, C., Mileham, A.J., McLaren, D.G., Whitelaw, C.B.A., Lillico, S.G., Highly efficient targeted chromosome deletions using CRISPR/Cas9. Biotechnol. Bioeng. 2015, 112, 1060-1064. 

Mammalian Cell Lines via CRISPR/Cas9. J. Vis. Exp. JoVE 2015.

[23] Xie, K., Minkenberg, B., Yang, Y., Boosting CRISPR/Cas9 multiplex editing capability with the endogenous tRNA-processing system. Proc. Natl. Acad. Sci. 2015, 112, 35703575.

[24] Nissim, L., Perli, S.D., Fridkin, A., Perez-Pinera, P., Lu, T.K., Multiplexed and Programmable Regulation of Gene Networks with an Integrated RNA and CRISPR/Cas Toolkit in Human Cells. Mol. Cell 2014, 54, 698-710.

[25] Gao, Y., Zhao, Y., Self-processing of ribozyme-flanked RNAs into guide RNAs in vitro and in vivo for CRISPR-mediated genome editing. J. Integr. Plant Biol. 2014, 56, 343349.

[26] Zetsche, B., Heidenreich, M., Mohanraju, P., Fedorova, I., Kneppers, J., DeGennaro, E.M., Winblad, N., Choudhury, S.R., Abudayyeh, O.O., Gootenberg, J.S., Wu, W.Y., Scott, D.A., Severinov, K., van der Oost, J., Zhang, F., Multiplex gene editing by CRISPR-Cpf1 using a single crRNA array. Nat. Biotechnol. 2016, advance online publication.

[27] Hsu, P.D., Scott, D.A., Weinstein, J.A., Ran, F.A., Konermann, S., Agarwala, V., Li, Y., Fine, E.J., Wu, X., Shalem, O., Cradick, T.J., Marraffini, L.A., Bao, G., Zhang, F., DNA targeting specificity of RNA-guided Cas9 nucleases. Nat. Biotechnol. 2013, 31, 827-832.

[28] Doench, J.G., Fusi, N., Sullender, M., Hegde, M., Vaimberg, E.W., Donovan, K.F., Smith, I., Tothova, Z., Wilen, C., Orchard, R., Virgin, H.W., Listgarten, J., Root, D.E., Optimized sgRNA design to maximize activity and minimize off-target effects of CRISPR-Cas9. Nat. Biotechnol. 2016, 34, 184-191.

[29] Livak, K.J., Schmittgen, T.D., Analysis of relative gene expression data using realtime quantitative PCR and the 2(-Delta Delta C(T)) Method. Methods San Diego Calif $2001,25,402-408$.

[30] Feichtinger, J., Hernández, I., Fischer, C., Hanscho, M., Auer, N., Hackl, M., Jadhav, V., Baumann, M., Krempl, P.M., Schmidl, C., Farlik, M., Schuster, M., Merkel, A., Sommer, A., Heath, S., Rico, D., Bock, C., Thallinger, G.G., Borth, N., Comprehensive genome and epigenome characterization of $\mathrm{CHO}$ cells in response to evolutionary pressures and over time. Biotechnol. Bioeng. 2016, n/a-n/a.

[31] Yamane-Ohnuki, N., Yamano, K., Satoh, M., Biallelic gene knockouts in Chinese hamster ovary cells. Methods Mol. Biol. Clifton NJ 2008, 435, 1-16.

[32] Shen, J.P., Zhao, D., Sasik, R., Luebeck, J., Birmingham, A., Bojorquez-Gomez, A., Licon, K., Klepper, K., Pekin, D., Beckett, A.N., Sanchez, K.S., Thomas, A., Kuo, C.-C., Du, D., Roguev, A., Lewis, N.E., Chang, A.N., Kreisberg, J.F., Krogan, N., Qi, L., Ideker, T., Mali, P., 
Combinatorial CRISPR-Cas9 screens for de novo mapping of genetic interactions. Nat. Methods 2017, advance online publication.

[33] Zhu, S., Li, W., Liu, J., Chen, C.-H., Liao, Q., Xu, P., Xu, H., Xiao, T., Cao, Z., Peng, J., Yuan, P., Brown, M., Liu, X.S., Wei, W., Genome-scale deletion screening of human long noncoding RNAs using a paired-guide RNA CRISPR-Cas9 library. Nat. Biotechnol. 2016, 34, 1279-1286.

[34] Port, F., Bullock, S.L., Augmenting CRISPR applications in Drosophila with tRNAflanked sgRNAs. Nat. Methods 2016, advance online publication.

[35] Port, F., Bullock, S.L., Expansion of the CRISPR toolbox in an animal with tRNAflanked Cas9 and Cpf1 gRNAs. bioRxiv 2016, 046417.

[36] Fagerlund, R.D., Staals, R.H.J., Fineran, P.C., The Cpf1 CRISPR-Cas protein expands genome-editing tools. Genome Biol. 2015, 16.

[37] Zhou, Y., Zhu, S., Cai, C., Yuan, P., Li, C., Huang, Y., Wei, W., High-throughput screening of a CRISPR/Cas9 library for functional genomics in human cells. Nature 2014, 509, 487-491.

[38] Konermann, S., Brigham, M.D., Trevino, A.E., Joung, J., Abudayyeh, O.O., Barcena, C., Hsu, P.D., Habib, N., Gootenberg, J.S., Nishimasu, H., Nureki, O., Zhang, F., Genome-scale transcriptional activation by an engineered CRISPR-Cas 9 complex. Nature 2015, 517, 583-588. 


\section{Figure legends}

Table 1. CRISPR/Cpf1 features in comparison to CRISPR/Cas9

\begin{tabular}{lcc}
\hline Category & Cas9 & Cpf1 \\
\hline Classification & class 2, type II & class 2, type V \\
Length & 96 bp sgRNA & 44 bp sgRNA \\
Activity & DNase & DNase \& RNase \\
PAM & 5'-NGG & 5'-TTN \\
DSB position & 3 nt upstream PAM & 17 nt downstream PAM \\
Cut & blunt-end & staggered \\
Off-target activity & ++ & + \\
Localization loop & 3'-end & 5'-end \\
\hline
\end{tabular}

A

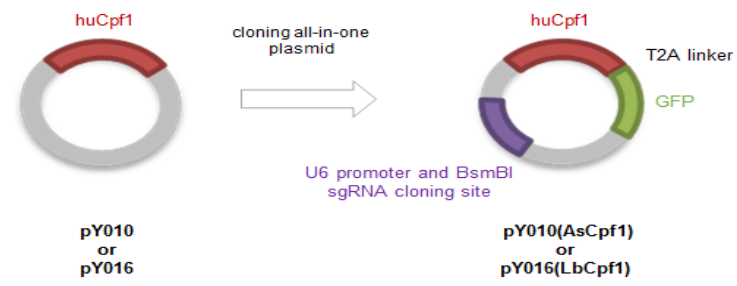

B

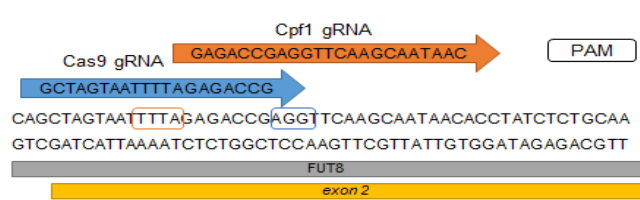

c
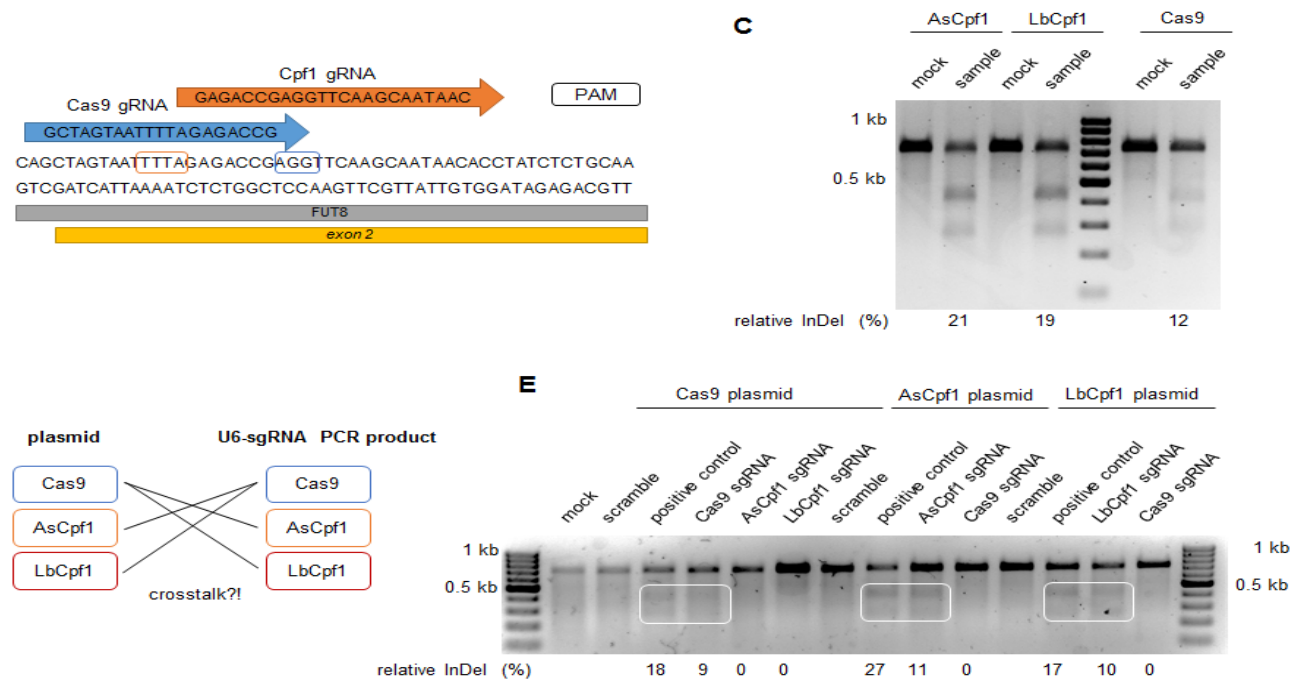

Figure 1. Analysis of CRISPR/Cpf1 activity in CHO-K1 and evaluation of crosstalk between Cas 9 and the two Cpf1 systems. (A) Illustration of all-in-one plasmid generation for AsCpf1 and LbCpf1. (B) FUT8 exon 2 genomic sequence. Cas9 and Cpf1 gRNA sequences are indicated with blue or orange arrows, respectively. PAM sequences are highlighted with boxes in the corresponding color. (C) Surveyor assay analysis of AsCpf1 and LbCpf1 activity in CHO-K1. Cleaved DNA fragments were detected as smaller bands than the unmodified mock signal. (D) Representation of the strategy to investigate potential crosstalk between Cas 9 and both Cpf1 systems. (E) Genome editing analysis by Surveyor assay to explore potential crosstalk between Cas 9 and both Cpf1 tools. 
A

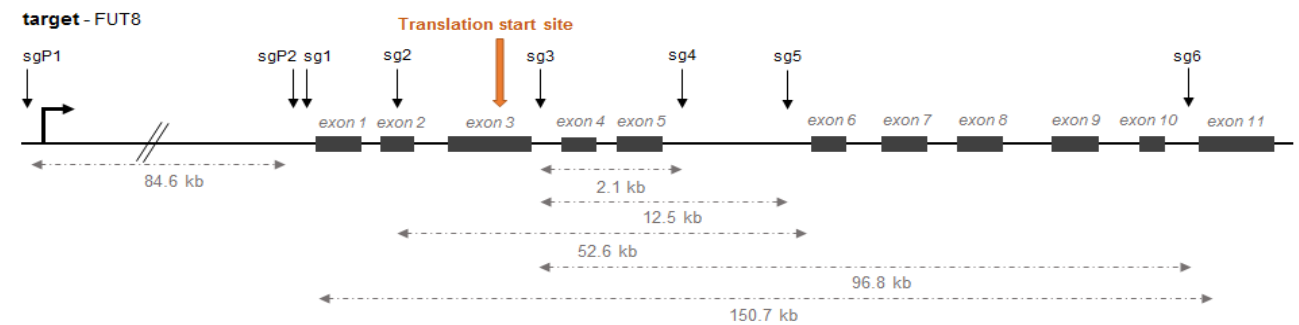

B
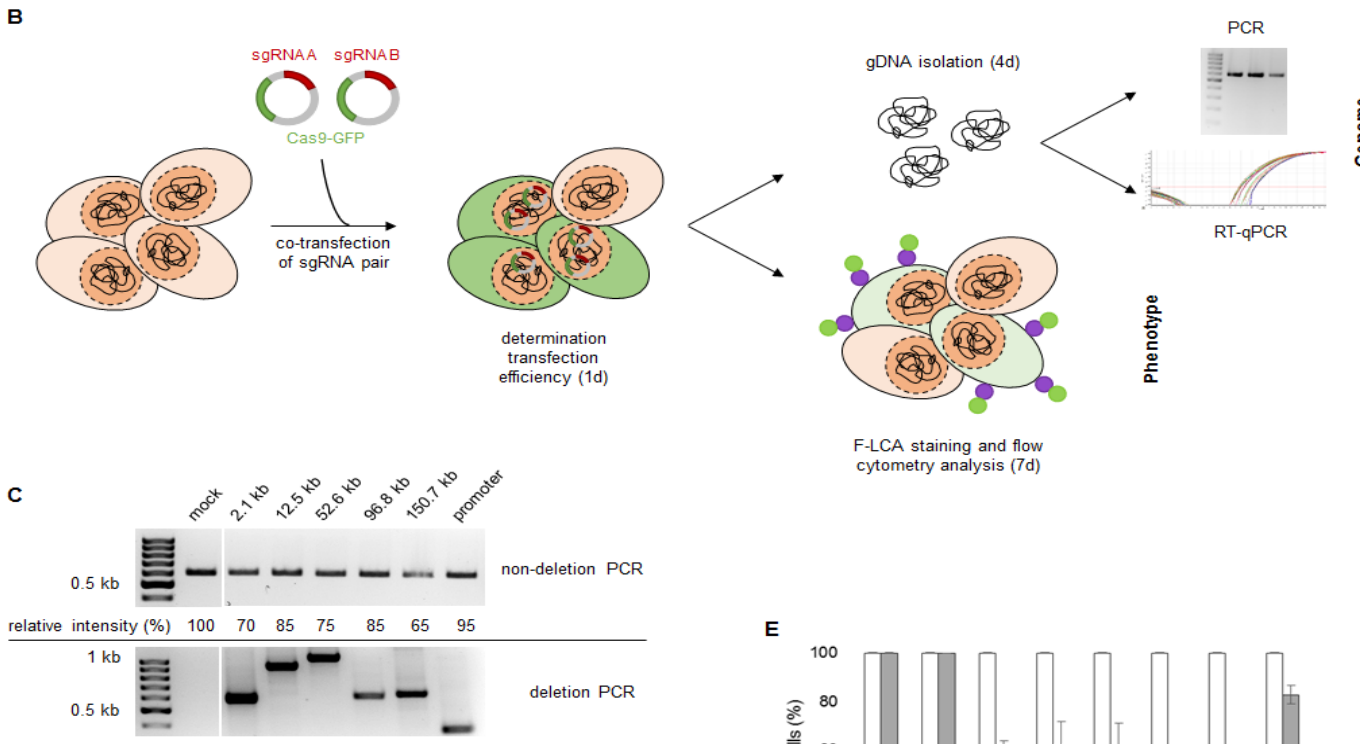

D
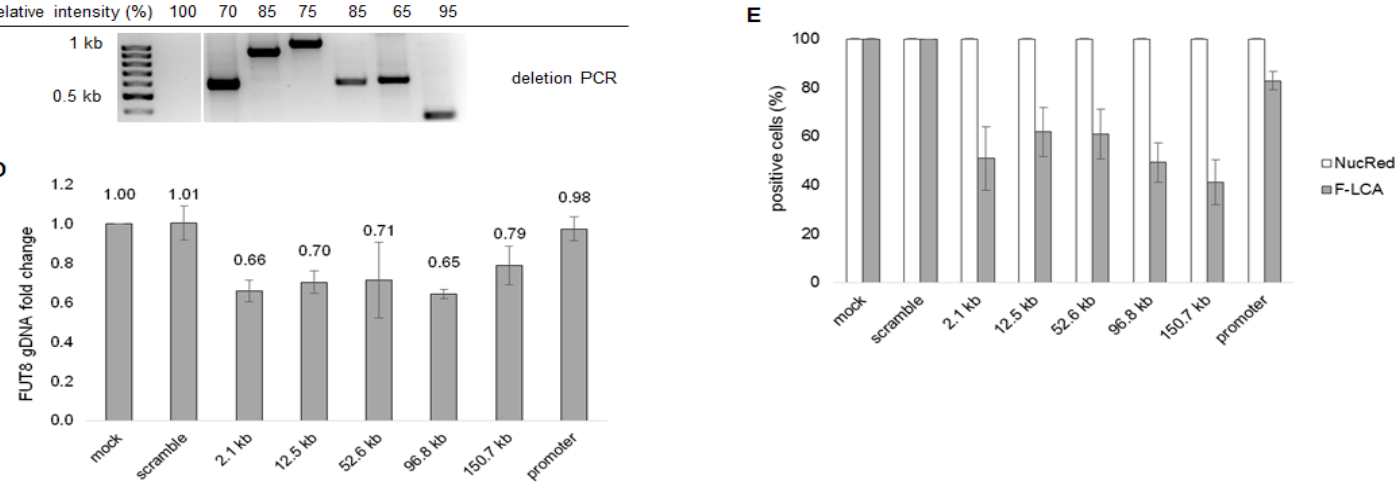

Figure 2. Efficiency of full genomic deletion of FUT8 by co-transfected CRISPR/Cas9 sgRNA pairs. (A) Schematic representation of the endogenous CHO-K1 FUT8 gene (160 kb). The promoter is depicted as a thick arrow. Target sites for all eight generated Cas9 sgRNAs in intergenic, intronic or untranslated exon regions are shown with black arrows and genomic deletion ranges are indicated with dotted lines. Translational start site is illustrated with an orange arrow. (B) Graphical workflow for the experimental setup to characterize generated genomic deletions, repeated in three biological replicates for each deletion size. (C) Nondeletion including relative decrease in signal intensities compared to mock control and deletion PCR results to identify the presence of genomic deletions (representative example of one biological replicate). (D) FUT8 gDNA level in modified CHO-K1 cell pools analyzed by qPCR, normalized against GUSB and related to the mock control (mean \pm standard deviation of three biological replicates, each in four technical replicates). (E) Phenotypical characterization of the modified cell pools by F-LCA and NucRed staining. F-LCA negative cells require a bi-allelic FUT8 deletion. NucRed staining detects live cells (mean \pm standard deviation of three biological replicates). 
A

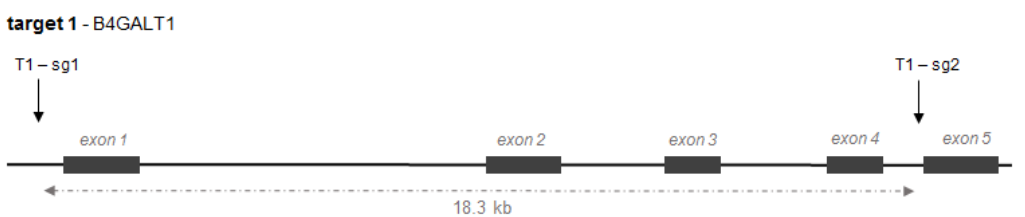

target 2 - B4GALT2

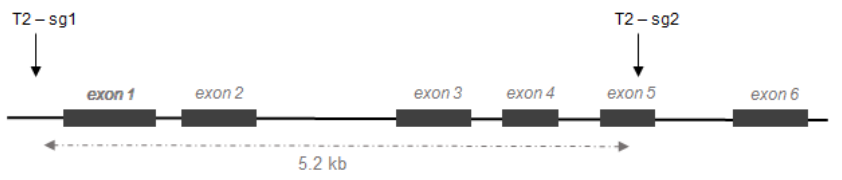

target 3 - B4GALT4
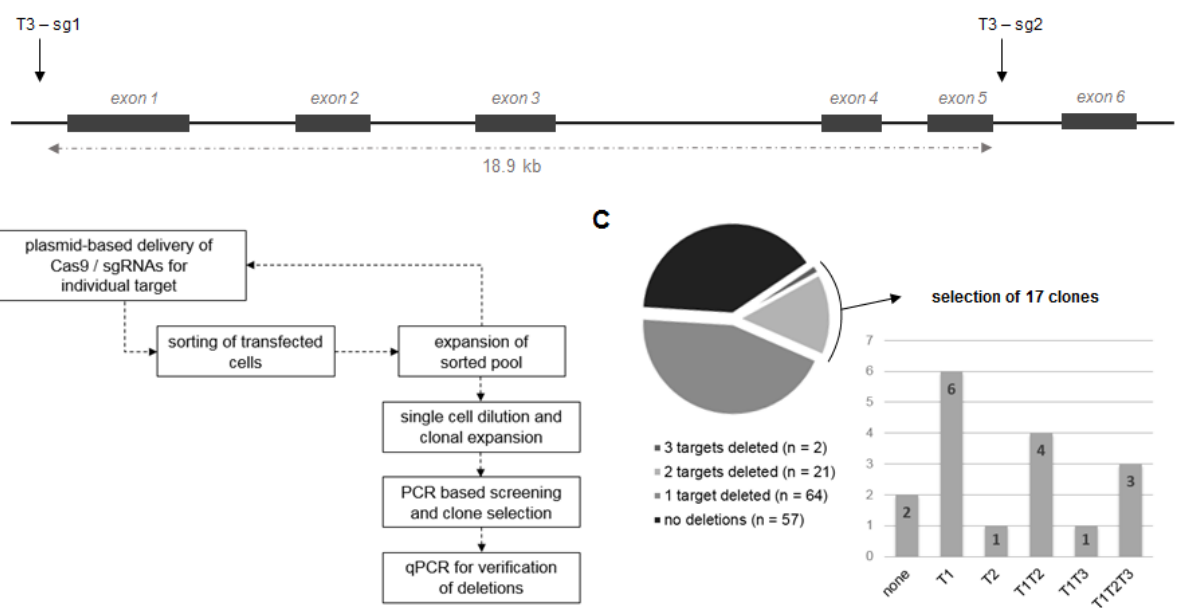

Figure 3. Generation of cell lines with multiple deletions in N-Glycosylation genes. (A) Schematic presentation of individual targets for deletion with indication of cut sites for selected sgRNA pairs. Fragments to be excised from B4GALT1, B4GALT2 and B4GALT4 are presented as dotted lines. (B) Overview of workflow including sequential rounds of transfection and sorting deleting only a single target at a time. (C) Results of pre-screening by non-deletion and deletion PCR are illustrated by the pie chart. Out of 23 clones showing profiles indicating triple or double deletions, 17 were subjected to further analysis by qPCR. The bar chart represents the final findings for clone characterization by qPCR. 
A

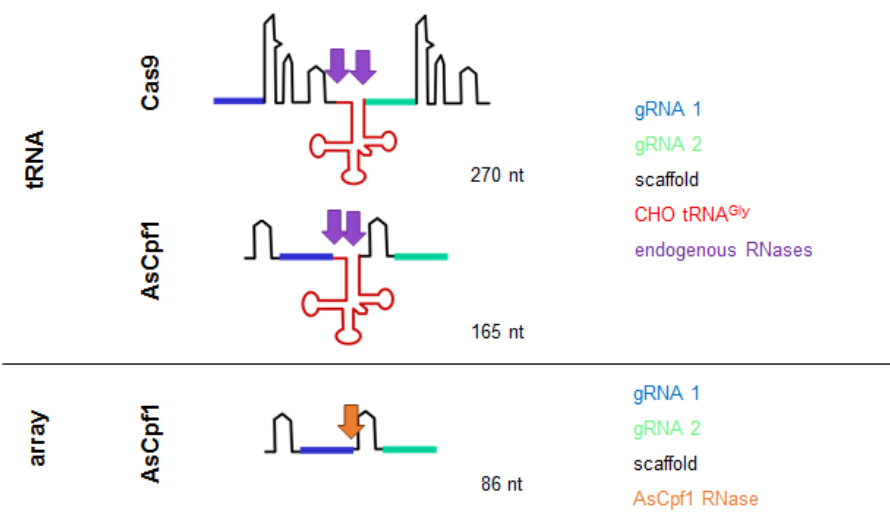

B

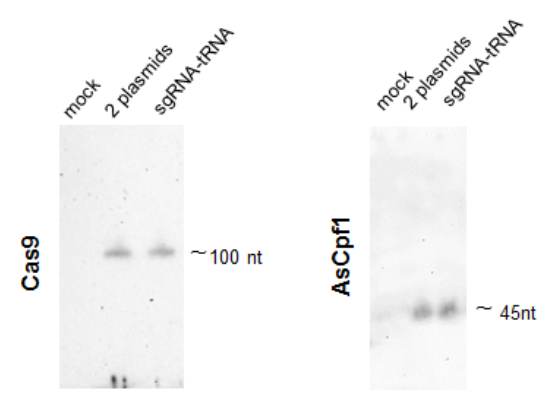

tRNA

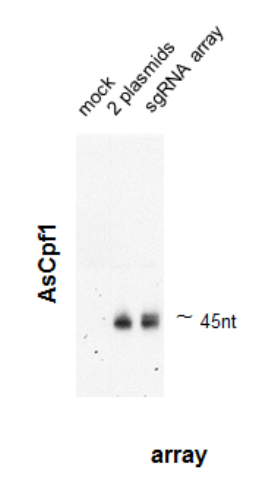

c

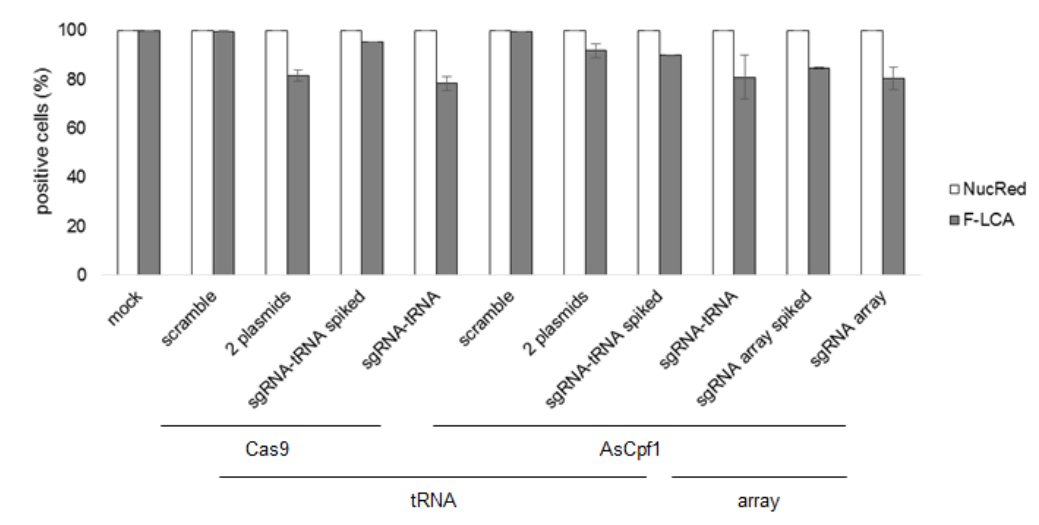

Figure 4. Strategies for bicistronic transcription of Cas 9 and AsCpf1 sgRNAs from a single promoter. (A) Illustrations of bicistronic sgRNA pairs for Cas9 and AsCpf1 using a CHO tRNA ${ }^{\text {Gly }}$ sequence as a linker (red). Positions for intracellular cleavage by endogenous RNases are indicated by purple arrows. For AsCpf1, a sgRNA array approach was additionally generated relying on the RNase activity of Cpf1 for processing at the site indicated by the orange arrow. (B) Northern Blot analysis of bicistronically transcribed Cas 9 and AsCpf1 sgRNAs using DIG-labeled probes against the Cas9 scaffold and one of the AsCpf1 sgRNAs. RNA from co-transfection with individual sgRNA plasmids was used as positive control. (C) Verification of the functionality of released Cas9 and AsCpf1 sgRNA pairs targeting FUT8 by phenotypical characterization of modified cell pools with F-LCA and NucRed staining (mean \pm standard deviation of three biological replicates). 